\title{
Cohen-Macaulay clutters with combinatorial optimization properties and parallelizations of normal edge ideals
}

\section{Luis A. Dupont ${ }^{1}$}

Departamento de Matemáticas, Centro de Investigación y de Estudios Avanzados del IPN

Apartado Postal 14-740, 07000 México City, D.F.

\section{Rafael H. Villarreal ${ }^{1}$}

Departamento de Matemáticas, Centro de Investigación y de Estudios Avanzados del IPN

Apartado Postal 14-740, 07000 México City, D.F.

E-mail address: vila@math.cinvestav.mx

\section{Enrique Reyes ${ }^{2}$}

Departamento de Ciencias Básicas, Unidad Profesional, Interdisciplinaria en Ingeniería y Tecnologias Avanzadas del IPN

UPIITA, Av. IPN 2580, Col. Barrio la Laguna Ticomán, 07340 México City, D.F.

\begin{abstract}
Let $\mathcal{C}$ be a uniform clutter and let $I=I(\mathcal{C})$ be its edge ideal. We prove that if $\mathcal{C}$ satisfies the packing property (resp. max-flow min-cut property), then there is a uniform Cohen-Macaulay clutter $\mathcal{C}_{1}$ satisfying the packing property (resp. max-flow min-cut property) such that $\mathcal{C}$ is a minor of $\mathcal{C}_{1}$. For arbitrary edge ideals of clutters we prove that the normality property is closed under parallelizations. Then we show some applications to edge ideals and clutters which are related to a conjecture of Conforti and Cornuéjols and to max-flow min-cut problems.

2000 Mathematics Subject Classification: Primary 13H10; Secondary 13F20, 13B22, 52B20.
\end{abstract}

\footnotetext{
${ }^{1}$ Partially supported by CONACyT grant 49251-F and SNI.

${ }^{2}$ Partially supported by COFAA-IPN and SNI.
} 


\section{Introduction}

Let $R=K\left[x_{1}, \ldots, x_{n}\right]$ be a polynomial ring over a field $K$ and let $I$ be an ideal of $R$ minimally generated by a finite set $F=\left\{x^{v_{1}}, \ldots, x^{v_{q}}\right\}$ of square-free monomials. As usual we use the notation $x^{a}:=x_{1}^{a_{1}} \cdots x_{n}^{a_{n}}$, where $a=\left(a_{1}, \ldots, a_{n}\right)$ is in $\mathbb{N}^{n}$. The support of a monomial $x^{a}$ is given by $\operatorname{supp}\left(x^{a}\right)=\left\{x_{i} \mid a_{i}>0\right\}$. For technical reasons we shall assume that each variable $x_{i}$ occurs in at least one monomial of $F$.

A clutter with finite vertex set $X$ is a family of subsets of $X$, called edges, none of which is included in another. The set of vertices of a clutter $\mathcal{C}$ is denoted by $V(\mathcal{C})$ and the set of edges of $\mathcal{C}$ is denoted by $E(\mathcal{C})$. A clutter is called $d$-uniform if all its edges have exactly $d$ vertices. We associate to the ideal $I$ a clutter $\mathcal{C}$ by taking the set of indeterminates $X=\left\{x_{1}, \ldots, x_{n}\right\}$ as vertex set and $E=\left\{S_{1}, \ldots, S_{q}\right\}$ as edge set, where $S_{k}$ is the support of $x^{v_{k}}$. The vector $v_{k}$ is called the characteristic vector of $S_{k}$. The assignment $I \mapsto \mathcal{C}$ gives a natural one to one correspondence between the family of square-free monomial ideals and the family of clutters. The ideal $I$ is called the edge ideal of $\mathcal{C}$. To stress the relationship between $I$ and $\mathcal{C}$ we will use the notation $I=I(\mathcal{C})$. Edge ideals of graphs were introduced and studied in $[24,29]$. Edge ideals of clutters also correspond to simplicial complexes via the Stanley-Reisner correspondence [25] and to facet ideals $[9,32]$. The Cohen-Macaulay property of edge ideals has been recently studied in $[3,10,16,22,27]$ using a combinatorial approach based on the notions of shellability, linear quotients, unmixedness, acyclicity and transitivity of digraphs, and the König property.

The aim of this note is to study the behavior, under certain operations, of various algebraic and combinatorial optimization properties of edge ideals and clutters such as the Cohen-Macaulay property, the normality, the torsion freeness, the packing and the max-flow min-cut properties. The study of edge ideals from the combinatorial optimization point of view was initiated in $[1,26]$ and continued in $[6,8,13,14,15,31]$, see also [17]. The Cohen-Macaulay and normality properties are two of the most interesting properties an edge ideal can have, see $[2,10,25,30]$ and $[19,28]$ respectively.

Recall that the integral closure of $I^{i}$, denoted by $\overline{I^{i}}$, is the ideal of $R$ given by

$$
\overline{I^{i}}=\left(\left\{x^{a} \in R \mid \exists p \geq 1 ;\left(x^{a}\right)^{p} \in I^{p i}\right\}\right) .
$$

An ideal $I$ is called normal if $I^{i}=\overline{I^{i}}$ for all $i$. A clutter obtained from $\mathcal{C}$ by a sequence of deletions and duplications of vertices is called a parallelization of $\mathcal{C}$ and a clutter obtained from $\mathcal{C}$ by a sequence of deletions and contractions of vertices is called a minor of $\mathcal{C}$, see Section 2. It is known that the normality of $I(\mathcal{C})$ is closed under minors [8]. One of our main results shows that the normality of $I(\mathcal{C})$ is closed under parallelizations: 
Theorem 2.3 Let $\mathcal{C}$ be a clutter and let $\mathcal{C}^{\prime}$ be a parallelization of $\mathcal{C}$. If $I(\mathcal{C})$ is normal, then $I\left(\mathcal{C}^{\prime}\right)$ is normal.

The ideal $I=I(\mathcal{C})$ is called normally torsion free if $I^{i}=I^{(i)}$ for all $i$, where $I^{(i)}$ is the $i t h$ symbolic power of $I$. As an application we prove that if $I(\mathcal{C})$ is normally torsion free and $\mathcal{C}^{\prime}$ is a parallelization of $\mathcal{C}$, then $I\left(\mathcal{C}^{\prime}\right)$ is normally torsion free (Corollary 2.12). Let $A$ be the incidence matrix of $\mathcal{C}$, i.e., $A$ is the matrix with column vectors $v_{1}, \ldots, v_{q}$. A clutter $\mathcal{C}$ satisfies the max-flow min-cut (MFMC) property if both sides of the LP-duality equation

$$
\min \{\langle w, x\rangle \mid x \geq 0 ; x A \geq \mathbf{1}\}=\max \{\langle y, \mathbf{1}\rangle \mid y \geq 0 ; A y \leq w\}
$$

have integral optimum solutions $x$ and $y$ for each non-negative integral vector $w$. A remarkable result of [15] (cf. [14, Theorem 4.6]) shows that $I(\mathcal{C})$ is normally torsion free if and only if $\mathcal{C}$ has the max-flow min-cut property. This fact makes a strong connection between commutative algebra and combinatorial optimization. It is known [23, Chapter 79] that a clutter $\mathcal{C}$ satisfies the max-flow min-cut property if and only if all parallelizations of the clutter $\mathcal{C}$ satisfy the König property (see Definition 2.7). As another application we give a proof of this fact using that the integrality of the polyhedron $\{x \mid x \geq 0 ; x A \geq \mathbf{1}\}$ is closed under parallelizations and minors and using that the normality of $I(\mathcal{C})$ is preserved under parallelizations and minors (Corollary 2.16).

A clutter $\mathcal{C}$ satisfies the packing property (PP for short) if all minors of $\mathcal{C}$ satisfy the König property. We say that a clutter $\mathcal{C}$ is Cohen-Macaulay if $R / I(\mathcal{C})$ is a Cohen-Macaulay ring, see [21]. The other main result of this note is:

Theorem 3.3 Let $\mathcal{C}$ be a d-uniform clutter on the vertex set $X$. Let

$$
Y=\left\{y_{i j} \mid 1 \leq i \leq n ; 1 \leq j \leq d-1\right\}
$$

be a set of new variables, and let $\mathcal{C}^{\prime}$ be the clutter with vertex set $V\left(\mathcal{C}^{\prime}\right)=$ $X \cup Y$ and edge set

$$
E\left(\mathcal{C}^{\prime}\right)=E(\mathcal{C}) \cup\left\{\left\{x_{1}, y_{11}, \ldots, y_{1(d-1)}\right\}, \ldots,\left\{x_{n}, y_{n 1}, \ldots, y_{n(d-1)}\right\}\right\} .
$$

Then the edge ideal $I\left(\mathcal{C}^{\prime}\right)$ is Cohen-Macaulay. If $\mathcal{C}$ satisfies PP (resp. maxflow min-cut), then $\mathcal{C}^{\prime}$ satisfies PP (resp. max-flow min-cut).

It is well known that if $\mathcal{C}$ satisfies the max-flow min-cut property, then $\mathcal{C}$ satisfies the packing property [5] (see Corollary 2.10). Conforti and Cornuéjols [4] conjecture that the converse is also true. Theorem 3.3 is interesting because it says that for uniform clutters it suffices to prove the conjecture for Cohen-Macaulay clutters, which have a rich structure. The 
Conforti-Cornuéjols conjecture has been studied in [7, 14, 15] using an algebraic approach based on certain algebraic properties of blowup algebras.

\section{Normality is preserved under parallelizations}

Let $\mathcal{C}$ be a clutter on the vertex set $X=\left\{x_{1}, \ldots, x_{n}\right\}$ and let $I=I(\mathcal{C})=$ $\left(x^{v_{1}}, \ldots, x^{v_{q}}\right)$ be its edge ideal. The incidence matrix of $\mathcal{C}$, denoted by $A=\left(a_{i j}\right)$, is the $n \times q$ matrix whose $(i, j)$ entry is given by $a_{i j}=1$ if $x_{i} \in g_{j}$ and $a_{i j}=0$ otherwise, where $g_{1}, \ldots, g_{q}$ are the edges of $\mathcal{C}$. Notice that the column vectors of $A$ are $v_{1}, \ldots, v_{q}$, where $v_{i}=\sum_{x_{j} \in g_{i}} e_{j}$ for $1 \leq i \leq q$. As usual $e_{j}$ denotes the $j$ th unit vector in $\mathbb{R}^{n}$. Recall that the Rees algebra of $I$ is given by:

$$
R[I t]:=R \oplus I t \oplus \cdots \oplus I^{i} t^{i} \oplus \cdots \subset R[t],
$$

where $t$ is a new variable. The Rees algebra of $I$ can be written as

$$
R[I t]=K\left[\left\{x^{a} t^{b} \mid(a, b) \in \mathbb{N} \mathcal{A}^{\prime}\right\}\right]
$$

where $\mathcal{A}^{\prime}=\left\{\left(v_{1}, 1\right), \ldots,\left(v_{q}, 1\right), e_{1}, \ldots, e_{n}\right\}$ and $\mathbb{N} \mathcal{A}^{\prime}$ is the subsemigroup of $\mathbb{N}^{n+1}$ spanned by $\mathcal{A}^{\prime}$. In other words $R[I t]$ is equal to $K\left[\mathbb{N} \mathcal{A}^{\prime}\right]$, the semigroup ring of $\mathbb{N} \mathcal{A}^{\prime}$, see [12]. On the other hand according to [30, Theorem 7.2.28] the integral closure of $R[I t]$ in its field of fractions can be expressed as

$$
\begin{aligned}
\overline{R[I t]} & =K\left[\left\{x^{a} t^{b} \mid(a, b) \in \mathbb{Z} \mathcal{A}^{\prime} \cap \mathbb{R}_{+} \mathcal{A}^{\prime}\right\}\right] \\
& =R \oplus \bar{I} t \oplus \overline{I^{2}} t^{2} \oplus \cdots \oplus \overline{I^{i}} t^{i} \oplus \cdots,
\end{aligned}
$$

where $\overline{I^{i}}$ is the integral closure of $I^{i}, \mathbb{R}_{+} \mathcal{A}^{\prime}$ is the cone spanned by $\mathcal{A}^{\prime}$, and $\mathbb{Z} \mathcal{A}^{\prime}$ is the subgroup spanned by $\mathcal{A}^{\prime}$. Notice that $\mathbb{Z} \mathcal{A}^{\prime}=\mathbb{Z}^{n+1}$. Hence $R[I t]$ is normal if and only if any of the following two equivalent conditions hold:

(a) $\mathbb{N} \mathcal{A}^{\prime} \equiv \mathbb{Z}^{n+1} \cap \mathbb{R}_{+} \mathcal{A}^{\prime}$.

(b) $I^{i}=\overline{I^{i}}$ for all $i \geq 1$.

If the second condition holds we say that $I$ is a normal ideal.

Let $\mathcal{C}$ be a clutter on the vertex set $X=\left\{x_{1}, \ldots, x_{n}\right\}$ and let $x_{i} \in X$. Then duplicating $x_{i}$ means extending $X$ by a new vertex $x_{i}^{\prime}$ and replacing $E(\mathcal{C})$ by

$$
E(\mathcal{C}) \cup\left\{\left(e \backslash\left\{x_{i}\right\}\right) \cup\left\{x_{i}^{\prime}\right\} \mid x_{i} \in e \in E(\mathcal{C})\right\} .
$$

The deletion of $x_{i}$, denoted by $\mathcal{C} \backslash\left\{x_{i}\right\}$, is the clutter formed from $\mathcal{C}$ by deleting the vertex $x_{i}$ and all edges containing $x_{i}$. A clutter obtained from $\mathcal{C}$ by a sequence of deletions and duplications of vertices is called a parallelization. If $w=\left(w_{i}\right)$ is a vector in $\mathbb{N}^{n}$, we denote by $\mathcal{C}^{w}$ the clutter obtained from $\mathcal{C}$ by deleting any vertex $x_{i}$ with $w_{i}=0$ and duplicating $w_{i}-1$ times any vertex $x_{i}$ if $w_{i} \geq 1$. The map $w \mapsto \mathcal{C}^{w}$ gives a one to one correspondence between $\mathbb{N}^{n}$ and the parallelizations of $\mathcal{C}$. 
Example 2.1. Let $G$ be the graph whose only edge is $\left\{x_{1}, x_{2}\right\}$ and let $w=(3,3)$. Then $G^{w}=\mathcal{K}_{3,3}$ is the complete bipartite graph with bipartition $V_{1}=\left\{x_{1}, x_{1}^{2}, x_{1}^{3}\right\}$ and $V_{2}=\left\{x_{2}, x_{2}^{2}, x_{2}^{3}\right\}$. Notice that $x_{i}^{k}$ is a vertex, i.e., $k$ is an index not an exponent.

The following notion of minor comes from combinatorial optimization $[4,23]$ and it is not apparently related to the minors (subdeterminants) of A.

Definition 2.2. Let $X^{\prime}=\left\{x_{i_{1}}, \ldots, x_{i_{r}}, x_{j_{1}}, \ldots, x_{j_{s}}\right\}$ be a subset of $X$. A minor of $I$ is a proper ideal $I^{\prime}$ of $R^{\prime}=K\left[X \backslash X^{\prime}\right]$ obtained from I by making $x_{i_{k}}=0$ and $x_{j_{\ell}}=1$ for all $k, \ell$. The ideal $I$ is considered itself a minor. $A$ minor of $\mathcal{C}$ is a clutter $\mathcal{C}^{\prime}$ whose edge ideal is $I^{\prime}$.

Notice that the generators of $I^{\prime}$ are obtained from the generators of $I$ by making $x_{i_{k}}=0$ and $x_{j_{\ell}}=1$ for all $k, \ell$. This means that $\mathcal{C}^{\prime}$ is obtained from $\mathcal{C}$ by shrinking some edges and deleting some other edges. Also notice that $\mathcal{C}^{\prime}$ is obtained from $I^{\prime}$ by considering the unique set of square-free monomials of $R^{\prime}$ that minimally generate $I^{\prime}$. If $I^{\prime}$ is the ideal obtained from $I$ by making $x_{i}=0$, then $I^{\prime}=I\left(\mathcal{C} \backslash\left\{x_{i}\right\}\right)$, i.e., making a variable equal to zero corresponds to a deletion. If $I^{\prime}$ is the minor obtained from $I$ by making $x_{i}=0$ for $1 \leq i \leq r$ and $x_{i}=1$ for $r+1 \leq i \leq s$, then in algebraic terms $I^{\prime}$ can be expressed as

$$
\left(I \cap K\left[x_{r+1}, \ldots, x_{n}\right]\right)_{\mathfrak{p}}=I^{\prime} K\left[x_{r+1}, \ldots, x_{n}\right]_{\mathfrak{p}},
$$

where $\left(I \cap K\left[x_{r+1}, \ldots, x_{n}\right]\right)_{\mathfrak{p}}$ and $K\left[x_{r+1}, \ldots, x_{n}\right]_{\mathfrak{p}}$ are localizations at the prime ideal $\mathfrak{p}$ generated by the variables $x_{s+1}, \ldots, x_{n}$.

It is known that the normality of $I(\mathcal{C})$ is closed under minors [8]. A main result of this section shows that the normality of $I(\mathcal{C})$ is closed under parallelizations.

Theorem 2.3. Let $\mathcal{C}$ be a clutter and let $\mathcal{C}^{\prime}$ be a parallelization of $\mathcal{C}$. If $I(\mathcal{C})$ is normal, then $I\left(\mathcal{C}^{\prime}\right)$ is normal.

Proof. From [8] we obtain that if $I(\mathcal{C})$ is normal and $\mathcal{C}^{\prime}$ is a minor of $\mathcal{C}$, then $I\left(\mathcal{C}^{\prime}\right)$ is also normal. Thus we need only show that the normality of $I(\mathcal{C})$ is preserved when we duplicate a vertex of $\mathcal{C}$. Let $V(\mathcal{C})=\left\{x_{2}, \ldots, x_{n}\right\}$ be the vertex set of $\mathcal{C}$ and let $\mathcal{C}^{\prime}$ be the clutter obtained from $\mathcal{C}$ by duplicating the vertex $x_{2}$, i.e., we extend $V(\mathcal{C})$ by a new vertex $x_{2}^{\prime}$ as explained before Example 2.1. For convenience we set $x_{1}=x_{2}^{\prime}$. We may assume that

$$
I=I(\mathcal{C})=\left(x_{2} x^{w_{1}}, \ldots, x_{2} x^{w_{r}}, x^{w_{r+1}}, \ldots, x^{w_{q}}\right),
$$

where $x^{w_{i}} \in K\left[x_{3}, \ldots, x_{n}\right]$ for all $i$. We must show that the ideal

$$
I\left(\mathcal{C}^{\prime}\right)=I+\left(x_{1} x^{w_{1}}, \ldots, x_{1} x^{w_{r}}\right)
$$


is normal. Consider the sets

$$
\begin{aligned}
\mathcal{A}= & \left\{e_{2}, \ldots, e_{n},\left(0,1, w_{1}, 1\right), \ldots,\left(0,1, w_{r}, 1\right),\left(0,0, w_{r+1}, 1\right), \ldots,\right. \\
& \left.\left(0,0, w_{q}, 1\right)\right\} \\
\mathcal{A}^{\prime}= & \mathcal{A} \cup\left\{e_{1},\left(1,0, w_{1}, 1\right), \ldots,\left(1,0, w_{r}, 1\right)\right\}
\end{aligned}
$$

By hypothesis $\mathbb{Z}^{n+1} \cap \mathbb{R}_{+} \mathcal{A}=\mathbb{N} \mathcal{A}$. We must prove that $\mathbb{Z}^{n+1} \cap \mathbb{R}_{+} \mathcal{A}^{\prime}=$ $\mathbb{N} \mathcal{A}^{\prime}$. It suffices to show that the left hand side is contained in the right hand side because the other inclusion always holds. Take an integral vector $(a, b, c, d)$ in $\mathbb{R}_{+} \mathcal{A}^{\prime}$, where $a, b, d \in \mathbb{Z}$ and $c \in \mathbb{Z}^{n-2}$. Then

$$
\begin{aligned}
(a, b, c, d) & =\sum_{i=1}^{r} \alpha_{i}\left(0,1, w_{i}, 1\right)+\sum_{i=r+1}^{q} \alpha_{i}\left(0,0, w_{i}, 1\right)+\sum_{i=1}^{r} \beta_{i}\left(1,0, w_{i}, 1\right) \\
& +\sum_{i=1}^{n} \gamma_{i} e_{i}
\end{aligned}
$$

for some $\alpha_{i}, \beta_{i}, \gamma_{i}$ in $\mathbb{R}_{+}$. Comparing entries one has

$$
\begin{aligned}
a & =\beta_{1}+\cdots+\beta_{r}+\gamma_{1}, \\
b & =\alpha_{1}+\cdots+\alpha_{r}+\gamma_{2}, \\
c & =\sum_{i=1}^{r}\left(\alpha_{i}+\beta_{i}\right) w_{i}+\sum_{i=r+1}^{q} \alpha_{i} w_{i}+\sum_{i=3}^{n} \gamma_{i} e_{i}, \\
d & =\sum_{i=1}^{r}\left(\alpha_{i}+\beta_{i}\right)+\sum_{i=r+1}^{q} \alpha_{i} .
\end{aligned}
$$

Consequently we obtain the equality

$$
\begin{aligned}
(0, a+b, c, d) & =\sum_{i=1}^{r}\left(\alpha_{i}+\beta_{i}\right)\left(0,1, w_{i}, 1\right)+\sum_{i=r+1}^{q} \alpha_{i}\left(0,0, w_{i}, 1\right)+\left(\gamma_{1}+\gamma_{2}\right) e_{2} \\
& +\sum_{i=3}^{n} \gamma_{i} e_{i},
\end{aligned}
$$

that is, the vector $(0, a+b, c, d)$ is in $\mathbb{Z}^{n+1} \cap \mathbb{R}_{+} \mathcal{A}=\mathbb{N} \mathcal{A}$. Thus there are $\lambda_{i}, \mu_{i}$ in $\mathbb{N}$ such that.

$$
(0, a+b, c, d)=\sum_{i=1}^{r} \mu_{i}\left(0,1, w_{i}, 1\right)+\sum_{i=r+1}^{q} \mu_{i}\left(0,0, w_{i}, 1\right)+\sum_{i=2}^{n} \lambda_{i} e_{i} .
$$


Comparing entries we obtain the equalities

$$
\begin{aligned}
a+b & =\mu_{1}+\cdots+\mu_{r}+\lambda_{2}, \\
c & =\mu_{1} w_{1}+\cdots+\mu_{q} w_{q}+\lambda_{3} e_{3}+\cdots+\lambda_{n} e_{n}, \\
d & =\mu_{1}+\cdots+\mu_{q} .
\end{aligned}
$$

Case (I): $b \leq \sum_{i=1}^{r} \mu_{i}$. If $b<\mu_{1}$, we set $b=\mu_{1}^{\prime}, \mu_{1}^{\prime}<\mu_{1}$, and define $\mu_{1}^{\prime \prime}=\mu_{1}-\mu_{1}^{\prime}$. Otherwise pick $s \geq 2$ such that

$$
\mu_{1}+\cdots+\mu_{s-1} \leq b \leq \mu_{1}+\cdots+\mu_{s}
$$

Then $b=\mu_{1}+\cdots+\mu_{s-1}+\mu_{s}^{\prime}$, where $\mu_{s}^{\prime} \leq \mu_{s}$. Set $\mu_{s}^{\prime \prime}=\mu_{s}-\mu_{s}^{\prime}$. Notice that

$$
\begin{aligned}
a+b & =\mu_{1}+\cdots+\mu_{r}+\lambda_{2}=a+\mu_{1}+\cdots+\mu_{s-1}+\mu_{s}^{\prime}, \\
a & =\mu_{s}+\cdots+\mu_{r}+\lambda_{2}-\mu_{s}^{\prime}=\mu_{s+1}+\cdots+\mu_{r}+\mu_{s}^{\prime \prime}+\lambda_{2} .
\end{aligned}
$$

Then

$$
\begin{aligned}
(a, b, c, d)= & \sum_{i=1}^{s-1} \mu_{i}\left(0,1, w_{i}, 1\right)+\mu_{s}^{\prime}\left(0,1, w_{s}, 1\right)+\sum_{i=r+1}^{q} \mu_{i}\left(0,0, w_{i}, 1\right) \\
& +\mu_{s}^{\prime \prime}\left(1,0, w_{s}, 1\right)+\sum_{i=s+1}^{r} \mu_{i}\left(1,0, w_{i}, 1\right)+\lambda_{2} e_{1}+\sum_{i=3}^{n} \lambda_{i} e_{i},
\end{aligned}
$$

that is, $(a, b, c, d) \in \mathbb{N} \mathcal{A}^{\prime}$.

Case (II): $b>\sum_{i=1}^{r} \mu_{i}$. Then $b=\sum_{i=1}^{r} \mu_{i}+\lambda_{2}^{\prime}$. Since

$$
a+b=\mu_{1}+\cdots+\mu_{r}+\lambda_{2}=a+\mu_{1}+\cdots+\mu_{r}+\lambda_{2}^{\prime}
$$

we get $a=\lambda_{2}-\lambda_{2}^{\prime}$. In particular $\lambda_{2} \geq \lambda_{2}^{\prime}$. Then

$$
(a, b, c, d)=\sum_{i=1}^{r} \mu_{i}\left(0,1, w_{i}, 1\right)+\sum_{i=r+1}^{q} \mu_{i}\left(0,0, w_{i}, 1\right)+a e_{1}+\lambda_{2}^{\prime} e_{2}+\sum_{i=3}^{n} \lambda_{i} e_{i}
$$

that is, $(a, b, c, d) \in \mathbb{N} \mathcal{A}^{\prime}$.

Our next goal is to present some applications of this result, but first we need to prove a couple of lemmas and we need to recall some notions and results.

Definition 2.4. A subset $C \subset X$ is a minimal vertex cover of the clutter $\mathcal{C}$ if: (i) every edge of $\mathcal{C}$ contains at least one vertex of $C$, and (ii) there is no proper subset of $C$ with the first property. If $C$ satisfies condition (i) only, then $C$ is called a vertex cover of $\mathcal{C}$. 
Definition 2.5. Let $A$ be the incidence matrix of $\mathcal{C}$. The clutter $\mathcal{C}$ satisfies the max-flow min-cut (MFMC) property if both sides of the LP-duality equation

$$
\min \{\langle w, x\rangle \mid x \geq 0 ; x A \geq \mathbf{1}\}=\max \{\langle y, \mathbf{1}\rangle \mid y \geq 0 ; A y \leq w\}
$$

have integral optimum solutions $x$ and $y$ for each non-negative integral vector $w$.

Let $A$ be the incidence matrix of $\mathcal{C}$ whose column vectors are $v_{1}, \ldots, v_{q}$. The set covering polyhedron of $\mathcal{C}$ is given by:

$$
Q(A)=\left\{x \in \mathbb{R}^{n} \mid x \geq 0 ; x A \geq \mathbf{1}\right\},
$$

where $\mathbf{1}=(1, \ldots, 1)$. This polyhedron was studied in $[14,15]$ to characterize the max-flow min-cut property of $\mathcal{C}$ and to study certain algebraic properties of blowup algebras. A clutter $\mathcal{C}$ is said to be ideal if $Q(A)$ is an integral polyhedron, i.e., it has only integral vertices. The integral vertices of $Q(A)$ are precisely the characteristic vectors of the minimal vertex covers of $\mathcal{C}$ [14, Proposition 2.2].

Theorem 2.6 ([8, 14, 15, 18]). The following conditions are equivalent:

(i) $g r_{I}(R)=R[I t] / I R[I t]$ is reduced, i.e., $g r_{I}(R)$ has no non-zero nilpotent elements.

(ii) $R[I t]$ is normal and $Q(A)$ is an integral polyhedron.

(iii) $I^{i}=I^{(i)}$ for $i \geq 1$, where $I^{(i)}$ is the ith symbolic power of $I$.

(iv) $\mathcal{C}$ has the max-flow min-cut property.

If condition (iii) is satisfied we say that $I$ is normally torsion free. A set of edges of the clutter $\mathcal{C}$ is independent or stable if no two of them have a common vertex. We denote the smallest number of vertices in any minimal vertex cover of $\mathcal{C}$ by $\alpha_{0}(\mathcal{C})$ and the maximum number of independent edges of $\mathcal{C}$ by $\beta_{1}(\mathcal{C})$. These numbers are related to min-max problems because they satisfy:

$$
\begin{aligned}
& \alpha_{0}(\mathcal{C}) \geq \min \{\langle\mathbf{1}, x\rangle \mid x \geq 0 ; x A \geq \mathbf{1}\} \\
& \quad=\max \{\langle y, \mathbf{1}\rangle \mid y \geq 0 ; A y \leq \mathbf{1}\} \geq \beta_{1}(\mathcal{C}) .
\end{aligned}
$$

Notice that $\alpha_{0}(\mathcal{C})=\beta_{1}(\mathcal{C})$ if and only if both sides of the equality have integral optimum solutions. These two numbers can be interpreted in terms of invariants of $I$. By [14] the height of the ideal $I$, denoted by $h t(I)$, is equal to the vertex covering number $\alpha_{0}(\mathcal{C})$ and the edge independence number $\beta_{1}(\mathcal{C})$ is equal to the maximum $r$ such that there exists a regular sequence of $r$ monomials inside $I$.

Definition 2.7. If $\alpha_{0}(\mathcal{C})=\beta_{1}(\mathcal{C})$ we say that the clutter $\mathcal{C}$ (or the ideal I) has the König property. 
Definition 2.8. The clutter $\mathcal{C}$ (or the ideal I) satisfy the packing property (PP for short) if all its minors satisfy the König property, i.e., $\alpha_{0}\left(\mathcal{C}^{\prime}\right)=$ $\beta_{1}\left(\mathcal{C}^{\prime}\right)$ for any minor $\mathcal{C}^{\prime}$ of $\mathcal{C}$.

Theorem 2.9. (A. Lehman; see [5, Theorem 1.8]) If $\mathcal{C}$ has the packing property, then $Q(A)$ is integral.

Corollary 2.10 ([5]). If the clutter $\mathcal{C}$ has the max-flow min-cut property, then $\mathcal{C}$ has the packing property.

Proof. Assume that the clutter $\mathcal{C}$ has the max-flow min-cut property. This property is closed under taking minors. Thus it suffices to prove that $\mathcal{C}$ has the König property. We denote the incidence matrix of $\mathcal{C}$ by $A$. By hypothesis the LP-duality equation

$$
\min \{\langle\mathbf{1}, x\rangle \mid x \geq 0 ; x A \geq \mathbf{1}\}=\max \{\langle y, \mathbf{1}\rangle \mid y \geq 0 ; A y \leq \mathbf{1}\}
$$

has optimum integral solutions $x, y$. To complete the proof notice that the left hand side of this equality is $\alpha_{0}(\mathcal{C})$ and the right hand side is $\beta_{1}(\mathcal{C})$.

Conforti and Cornuéjols conjecture that the converse is also true:

Conjecture 2.11. ([4]) If the clutter $\mathcal{C}$ has the packing property, then $\mathcal{C}$ has the max-flow min-cut property.

To the best of our knowledge this conjecture is open, see [5, Conjecture 1.6].

Corollary 2.12. Let $\mathcal{C}$ be a clutter and let $\mathcal{C}^{\prime}$ be a parallelization of $\mathcal{C}$. If $I(\mathcal{C})$ is normally torsion free, then $I\left(\mathcal{C}^{\prime}\right)$ is normally torsion free.

Proof. Let $A$ and $A^{\prime}$ be the incidence matrices of $\mathcal{C}$ and $\mathcal{C}^{\prime}$ respectively. By Theorem 2.6 the ideal $I(\mathcal{C})$ is normal and $Q(A)$ is integral. From Theorem 2.3 the ideal $I\left(\mathcal{C}^{\prime}\right)$ is normal, and since the integrality of $Q(A)$ is closed under minors and parallelizations (see [14] and [23]) we get that $Q\left(A^{\prime}\right)$ is again integral. Thus applying Theorem 2.6 once more we get that $I\left(\mathcal{C}^{\prime}\right)$ is normally torsion free.

Corollary 2.13. Let $\mathcal{C}$ be a clutter and let $\mathcal{C}^{\prime}$ be a parallelization of $\mathcal{C}$. If $\mathcal{C}$ has the max-flow min-cut property, then $\mathcal{C}^{\prime}$ has the König property. In particular $\mathcal{C}^{w}$ has the König property for all $w \in \mathbb{N}^{n}$.

Proof. By Corollary 2.12 the clutter $\mathcal{C}^{\prime}$ has the max-flow min-cut property. Thus applying Corollary 2.10 we obtain that $\mathcal{C}^{\prime}$ has the König property.

Lemma 2.14. Let $\mathcal{C}$ be a clutter and let $A$ be its incidence matrix. If $w=\left(w_{i}\right)$ is a vector in $\mathbb{N}^{n}$, then

$$
\beta_{1}\left(\mathcal{C}^{w}\right) \leq \max \left\{\langle y, \mathbf{1}\rangle \mid y \in \mathbb{N}^{q} ; A y \leq w\right\}
$$


Proof. We may assume that $w=\left(w_{1}, \ldots, w_{m}, 0, \ldots, 0\right)$, where $w_{i} \geq 1$ for $i=1, \ldots, m$. Recall that for each $i$ the vertex $x_{i}$ is duplicated $w_{i}-1$ times. We denote the duplications of $x_{i}$ by $x_{i}^{2}, \ldots, x_{i}^{w_{i}}$ and set $x_{i}^{1}=x_{i}$. Thus the vertex set of $\mathcal{C}^{w}$ is equal to

$$
V\left(\mathcal{C}^{w}\right)=\left\{x_{1}^{1}, \ldots, x_{1}^{w_{1}}, \ldots, x_{i}^{1}, \ldots, x_{i}^{w_{i}}, \ldots, x_{m}^{1}, \ldots, x_{m}^{w_{m}}\right\} .
$$

There are $f_{1}, \ldots, f_{\beta_{1}}$ independent edges of $\mathcal{C}^{w}$, where $\beta_{1}=\beta_{1}\left(\mathcal{C}^{w}\right)$. Each $f_{i}$ has the form

$$
f_{k}=\left\{x_{k_{1}}^{j_{k_{1}}}, x_{k_{2}}^{j_{k_{2}}}, \ldots, x_{k_{r}}^{j_{k_{r}}}\right\} \quad\left(1 \leq k_{1}<\cdots<k_{r} \leq m ; 1 \leq j_{k_{i}} \leq w_{k_{i}}\right) .
$$

We set $g_{k}=\left\{x_{k_{1}}^{1}, x_{k_{2}}^{1}, \ldots, x_{k_{r}}^{1}\right\}=\left\{x_{k_{1}}, x_{k_{2}}, \ldots, x_{k_{r}}\right\}$. By definition of $\mathcal{C}^{w}$ we get that $g_{k} \in E(\mathcal{C})$ for all $k$. We may re-order the $f_{i}$ so that

$$
\underbrace{g_{1}=g_{2}=\cdots=g_{s_{1}}}_{s_{1}}, \underbrace{g_{s_{1}+1}=\cdots=g_{s_{2}}}_{s_{2}-s_{1}}, \ldots, \underbrace{g_{s_{r-1}+1}=\cdots=g_{s_{r}}}_{s_{r}-s_{r-1}}
$$

and $g_{s_{1}}, \ldots, g_{s_{r}}$ distinct, where $s_{r}=\beta_{1}$. Let $v_{i}$ be the characteristic vector of $g_{s_{i}}$. Set $y=s_{1} e_{1}+\left(s_{2}-s_{1}\right) e_{2}+\cdots+\left(s_{r}-s_{r-1}\right) e_{r}$. We may assume that the incidence matrix $A$ of $\mathcal{C}$ has column vector $v_{1}, \ldots, v_{q}$. Then $y$ satisfies $\langle y, \mathbf{1}\rangle=\beta_{1}$. For each $k_{i}$ the number of variables of the form $x_{k_{i}}^{\ell}$ that occur in $f_{1}, \ldots, f_{\beta_{1}}$ is at most $w_{k_{i}}$ because the $f_{i}$ are pairwise disjoint. Hence for each $k_{i}$ the number of times that the variable $x_{k_{i}}^{1}$ occurs in $g_{1}, \ldots, g_{\beta_{1}}$ is at most $w_{k_{i}}$. Then

$$
A y=s_{1} v_{1}+\left(s_{2}-s_{1}\right) v_{2}+\cdots+\left(s_{r}-s_{r-1}\right) v_{r} \leq w .
$$

Therefore we obtain the required inequality.

Let $\mathcal{C}$ be a clutter. For use below we denote the set of minimal vertex covers of $\mathcal{C}$ by $\Upsilon(\mathcal{C})$.

Lemma 2.15. Let $\mathcal{C}$ be a clutter and let $A$ be its incidence matrix. If $w=\left(w_{i}\right)$ is a vector in $\mathbb{N}^{n}$, then

$$
\min \left\{\sum_{x_{i} \in C} w_{i} \mid C \in \Upsilon(\mathcal{C})\right\}=\alpha_{0}\left(\mathcal{C}^{w}\right) .
$$

Proof. We may assume that $w=\left(w_{1}, \ldots, w_{m}, w_{m+1}, \ldots, w_{m_{1}}, 0, \ldots, 0\right)$, where $w_{i} \geq 2$ for $i=1, \ldots, m, w_{i}=1$ for $i=m+1, \ldots, m_{1}$, and $w_{i}=0$ for $i>m_{1}$. Thus for $i=1, \ldots, m$ the vertex $x_{i}$ is duplicated $w_{i}-1$ times. We denote the duplications of $x_{i}$ by $x_{i}^{2}, \ldots, x_{i}^{w_{i}}$ and set $x_{i}^{1}=x_{i}$.

We first prove that the left hand side is less or equal than the right hand side. Let $C$ be a minimal vertex cover of $\mathcal{C}^{w}$ with $\alpha_{0}$ elements, where $\alpha_{0}=\alpha_{0}\left(\mathcal{C}^{w}\right)$. We may assume that $C \cap\left\{x_{1}, \ldots, x_{m_{1}}\right\}=\left\{x_{1}, \ldots, x_{s}\right\}$. Note 
that $x_{i}^{1}, \ldots, x_{i}^{w_{i}}$ are in $C$ for $i=1, \ldots, s$. Indeed since $C$ is a minimal vertex cover of $\mathcal{C}^{w}$, there exists an edge $e$ of $\mathcal{C}^{w}$ such that $e \cap C=\left\{x_{i}^{1}\right\}$. Then $\left(e \backslash\left\{x_{i}^{1}\right\}\right) \cup\left\{x_{i}^{j}\right\}$ is an edge of $\mathcal{C}^{w}$ for $j=1, \ldots, w_{i}$. Consequently $x_{i}^{j} \in C$ for $j=1, \ldots, w_{i}$. Hence

$$
w_{1}+\cdots+w_{s} \leq|C|=\alpha_{0} .
$$

On the other hand the set $C^{\prime}=\left\{x_{1}, \ldots, x_{s}\right\} \cup\left\{x_{m_{1}+1}, \ldots, x_{n}\right\}$ is a vertex cover of $\mathcal{C}$. Let $D$ be a minimal vertex cover of $\mathcal{C}$ contained in $C^{\prime}$. Let $e_{D}$ denote the characteristic vector of $D$. Then, since $w_{i}=0$ for $i>m_{1}$, using Eq. (2) we get

$$
\left\langle w, e_{D}\right\rangle=\sum_{x_{i} \in D} w_{i}=\sum_{x_{i} \in D \cap\left\{x_{1}, \ldots, x_{s}\right\}} w_{i} \leq \sum_{x_{i} \in\left\{x_{1}, \ldots, x_{s}\right\}} w_{i} \leq \alpha_{0} .
$$

This completes the proof of the asserted inequality.

Next we show that the right hand side of the inequality is less or equal than the left hand side. Let $C$ be a minimal vertex cover of $\mathcal{C}$. Note that the set

$$
C^{\prime}=\cup_{x_{i} \in C}\left\{x_{i}^{1}, \ldots, x_{i}^{w_{i}}\right\}
$$

is a vertex cover of $\mathcal{C}^{w}$. Indeed any edge $e^{w}$ of $\mathcal{C}^{w}$ has the form $e^{w}=$ $\left\{x_{i_{1}}^{j_{1}}, \ldots, x_{i_{r}}^{j_{r}}\right\}$ for some edge $e=\left\{x_{i_{1}}, \ldots, x_{i_{r}}\right\}$ of $\mathcal{C}$ and since $e$ is covered by $C$, we have that $e^{w}$ is covered by $C^{\prime}$. Hence $\alpha_{0}\left(\mathcal{C}^{w}\right) \leq\left|C^{\prime}\right|=\sum_{x_{i} \in C} w_{i}$. As $C$ was an arbitrary vertex cover of $\mathcal{C}$ we get the asserted inequality.

Corollary 2.16. [23, Chapter 79] Let $\mathcal{C}$ be a clutter. Then $\mathcal{C}$ satisfies the max-flow min-cut property if and only if $\beta_{1}\left(\mathcal{C}^{w}\right)=\alpha_{0}\left(\mathcal{C}^{w}\right)$ for all $w \in \mathbb{N}^{n}$.

Proof. If $\mathcal{C}$ has the max-flow min-cut property, then $\mathcal{C}^{w}$ has the König property by Corollary 2.13. Conversely if $\mathcal{C}^{w}$ has the König property for all $w \in \mathbb{N}^{n}$, then by Lemmas 2.14 and 2.15 both sides of the LP-duality equation

$$
\min \{\langle w, x\rangle \mid x \geq 0 ; x A \geq \mathbf{1}\}=\max \{\langle y, \mathbf{1}\rangle \mid y \geq 0 ; A y \leq w\}
$$

have integral optimum solutions $x$ and $y$ for each non-negative integral vector $w$, i.e., $\mathcal{C}$ has the max-flow min-cut property.

\section{Cohen-Macaulay ideals with max-flow min-cut}

One of the aims here is to show how to construct Cohen-Macaulay clutters satisfying max-flow min-cut, $\mathrm{PP}$, and normality properties. Let $\mathcal{C}$ be a uniform clutter. A main result of this section proves that if $\mathcal{C}$ satisfies PP (resp. max-flow min-cut), then there is a uniform Cohen-Macaulay clutter $\mathcal{C}_{1}$ satisfying PP (resp. max-flow min-cut) such that $\mathcal{C}$ is a minor 
of $\mathcal{C}_{1}$. In particular for uniform clutters we prove that it suffices to show Conjecture 2.11 for Cohen-Macaulay clutters (see Corollary 3.4).

Let $R=K\left[x_{1}, \ldots, x_{n}\right]$ be a polynomial ring over a field $K$ and let $\mathcal{C}$ be a clutter on the vertex set $X$. As usual, in what follows, we denote the edge ideal of $\mathcal{C}$ by $I=I(\mathcal{C})$. Recall that $\mathfrak{p}$ is a minimal prime of $I=I(\mathcal{C})$ if and only if $\mathfrak{p}=(C)$ for some minimal vertex cover $C$ of $\mathcal{C}[30$, Proposition 6.1.16]. Thus the primary decomposition of the edge ideal of $\mathcal{C}$ is given by

$$
I(\mathcal{C})=\left(C_{1}\right) \cap\left(C_{2}\right) \cap \cdots \cap\left(C_{p}\right),
$$

where $C_{1}, \ldots, C_{p}$ are the minimal vertex covers of $\mathcal{C}$. In particular observe that the height of $I(\mathcal{C})$, denoted by $h t I(\mathcal{C})$, is equal to the minimum cardinality of a minimal vertex cover of $\mathcal{C}$. Also notice that the associated primes of $I(\mathcal{C})$ are precisely the minimal primes of $I(\mathcal{C})$.

Proposition 3.1. Let $R\left[z_{1}, \ldots, z_{\ell}\right]$ be a polynomial ring over $R$. If $I$ is a normal ideal of $R$, then $J=\left(I, x_{1} z_{1} \cdots z_{\ell}\right)$ is a normal ideal of $R\left[z_{1}, \ldots, z_{\ell}\right]$.

Proof. By induction on $p$ we will show $\overline{J^{p}}=J^{p}$ for all $p \geq 1$. If $p=1$, then $\bar{J}=J$ because $J$ is square-free (see [30, Corollary 7.3.15]). Assume $\overline{J^{i}}=J^{i}$ for $i<p$ and $p \geq 2$. Let $y$ be a monomial in $\overline{J^{p}}$, then $y^{m} \in J^{p m}$, for some $m>0$. Since $\overline{J^{p}} \subset \overline{J^{p-1}}=J^{p-1}$ we can write

$$
y=z_{1}^{t_{1}} \cdots z_{\ell}^{t_{\ell}}\left(x_{1} z_{1} \cdots z_{\ell}\right)^{r} M f_{1} \cdots f_{p-r-1},
$$

where $M$ is a monomial with $z_{i} \notin \operatorname{supp}(M)$ for all $i$ and the $f_{i}$ 's are monomials in $J$ with $z_{i} \notin \operatorname{supp}\left(f_{j}\right)$ for all $i, j$. We set $h=M f_{1} \cdots f_{p-r-1}$. It suffices to show that $y \in J^{p}$. Since $y^{m} \in J^{p m}$ we have

$$
y^{m}=z_{1}^{m t_{1}} \cdots z_{\ell}^{m t_{\ell}}\left(x_{1} z_{1} \cdots z_{\ell}\right)^{r m} h^{m}=N\left(x_{1} z_{1} \cdots z_{\ell}\right)^{s} g_{1} \cdots g_{m p-s},
$$

where $N$ is a monomial, $z_{i} \notin \operatorname{supp}\left(g_{j}\right)$ for all $i, j$, and the $g_{i}$ 's are monomials in $J$. We distinguish two cases:

Case (a): Assume $t_{i}=0$ for some $i$, then $s \leq r m$ because $z_{i}^{r m}$ is the maximum power of $z_{i}$ that divides $y^{m}$. Making $z_{j}=1$ for $j=1, \ldots, \ell$ in Eq. (3) we get

$$
x_{1}^{r m-s} h^{m}=N^{\prime} g_{1} \cdots g_{m p-s} .
$$

Thus $h^{m} \in I^{(m p-s)-(r m-s)}=I^{m(p-r)}$. Therefore we get $h \in \overline{I^{p-r}}=I^{p-r}$ and $y=z_{1}^{t_{1}} \cdots z_{\ell}^{t_{\ell}}\left(x_{1} z_{1} \cdots z_{\ell}\right)^{r} h \in J^{p}$.

Case (b): If $t_{i}>0$ for all $i$, we may assume $x_{1} \notin \operatorname{supp}(M)$, otherwise $y \in J^{p}$. We may also assume $x_{1} \notin \operatorname{supp}\left(f_{i}\right)$ for all $i$, otherwise it is not hard to see that we are back in case (a). Notice that $s \leq r m$, because $x_{1} \notin \operatorname{supp}(h)$. From Eq. (3) it follows that $h \in \overline{I^{p-r}}=I^{p-r}$ and $y=$ $z_{1}^{t_{1}} \cdots z_{\ell}^{t_{\ell}}\left(x_{1} z_{1} \cdots z_{\ell}\right)^{r} h \in J^{p}$. 
Lemma 3.2. Let $R\left[z_{1}, \ldots, z_{\ell}\right]$ be a polynomial ring over $R$ and let $I_{1}$ be the ideal obtained from $I$ by making $x_{1}=0$. Then: (a) if $I$ and $I_{1}$ satisfy the König property, then the ideal $J=\left(I, x_{1} z_{1} \cdots z_{\ell}\right)$ satisfies the König property, and (b) if I satisfies $P P$, then $J$ satisfies $P P$.

Proof. (a): If $h t(I)=h t(J)$, then $J$ satisfies König because $I$ does. Assume that $g=h t(I)<h t(J)$. Then $h t(J)=g+1$. Notice that every associated prime ideal of $I$ of height $g$ cannot contain $x_{1}$. We claim that $h t\left(I_{1}\right)=g$. If $r=h t\left(I_{1}\right)<g$, pick a minimal prime $\mathfrak{p}$ of $I_{1}$ of height $r$. Then $\mathfrak{p}+\left(x_{1}\right)$ is a prime ideal of height at most $g$ containing both $I$ and $x_{1}$, a contradiction. This proves the claim. Since $I_{1}$ satisfies König, there are $g$ independent monomials in $I_{1}$. Hence $h_{1}, \ldots, h_{g}, x_{1} z_{1} \cdots z_{\ell}$ are $g+1$ independent monomials in $J$, as required. Part (b) follows readily from part (a).

Theorem 3.3. Let $\mathcal{C}$ be a d-uniform clutter on the vertex set $X$. Let

$$
Y=\left\{y_{i j} \mid 1 \leq i \leq n ; 1 \leq j \leq d-1\right\}
$$

be a set of new variables, and let $\mathcal{C}^{\prime}$ be the clutter with vertex set $V\left(\mathcal{C}^{\prime}\right)=$ $X \cup Y$ and edge set

$$
E\left(\mathcal{C}^{\prime}\right)=E(\mathcal{C}) \cup\left\{\left\{x_{1}, y_{11}, \ldots, y_{1(d-1)}\right\}, \ldots,\left\{x_{n}, y_{n 1}, \ldots, y_{n(d-1)}\right\}\right\} .
$$

Then the edge ideal $I\left(\mathcal{C}^{\prime}\right)$ is Cohen-Macaulay. If $\mathcal{C}$ satisfies PP (resp. maxflow min-cut), then $\mathcal{C}^{\prime}$ satisfies PP (resp. max-flow min-cut).

Proof. Set $S=K[X \cup Y]$ and $I^{\prime}=I\left(\mathcal{C}^{\prime}\right)$. The clutter $\mathcal{C}^{\prime}$ is a grafting of $\mathcal{C}$ as defined by Faridi in [10]. Then $I^{\prime}$ is Cohen-Macaulay by [10, Theorem 8.2]. If $\mathcal{C}$ satisfies PP, then from Lemma 3.2(b) it follows that $\mathcal{C}^{\prime}$ satisfies PP. Assume that $\mathcal{C}$ satisfies MFMC. By Proposition $3.1 S\left[I^{\prime} t\right]$ is normal. Since $\mathcal{C}^{\prime}$ satisfies PP, by Lehman's theorem we get that $Q\left(A^{\prime}\right)$ is integral, where $A^{\prime}$ is the incidence matrix of $\mathcal{C}^{\prime}$. Therefore using Theorem 2.6 we conclude that $\mathcal{C}^{\prime}$ has MFMC.

Recall that a clutter $\mathcal{C}$ is called Cohen-Macaulay (CM for short) if $R / I(\mathcal{C})$ is a Cohen-Macaulay ring. Since $\mathcal{C}$ is a minor of $\mathcal{C}^{\prime}$ we obtain:

Corollary 3.4. Let $\mathcal{C}$ be a uniform clutter. If $\mathcal{C}$ satisfies PP (resp. maxflow min-cut), then there is a uniform Cohen-Macaulay clutter $\mathcal{C}_{1}$ satisfying $P P\left(\right.$ resp. max-flow min-cut) such that $\mathcal{C}$ is a minor of $\mathcal{C}_{1}$.

This result is interesting because it says that for uniform clutters it suffices to prove Conjecture 2.11 for Cohen-Macaulay clutters, which have a rich structure. 


\section{References}

[1] V. Bonanzinga, C. Escobar and R. H. Villarreal, On the normality of Rees algebras associated to totally unimodular matrices, Results Math. 41, 3/4, (2002), 258-264.

[2] W. Bruns and J. Herzog, Cohen-Macaulay Rings, Cambridge University Press, Cambridge, Revised Edition, 1997.

[3] G. Carrá Ferro and D. Ferrarello, Cohen-Macaulay graphs arising from digraphs. (2007) Preprint. math.AC/0703417.

[4] M. Conforti and G. Cornuéjols, Clutters that pack and the Max-Flow Min-Cut property: A conjecture, The Fourth Bellairs Workshop on Combinatorial Optimization (W. R. Pulleyblank, F. B. Shepherd, eds.).

[5] G. Cornuéjols, Combinatorial optimization: Packing and covering, CBMS-NSF Regional Conference Series in Applied Mathematics 74, SIAM (2001).

[6] D. Delfino, A. Taylor, W. V. Vasconcelos, R. H. Villarreal and N. Weininger, Monomial ideals and the computation of multiplicities, Commutative ring theory and applications (Fez, 2001), pp. 87-106, Lecture Notes in Pure and Appl. Math. 231, Dekker, New York, 2003.

[7] L. Dupont and R. H. Villarreal, Algebraic and combinatorial properties of ideals and algebras of uniform clutters of TDI systems, arXiv:math.AC/08011478, 2008.

[8] C. Escobar, R. H. Villarreal and Y. Yoshino, Torsion freeness and normality of blowup rings of monomial ideals, Commutative Algebra, Lect. Notes Pure Appl. Math. 244, Chapman \& Hall/CRC, Boca Raton, FL, 2006, pp. 69-84.

[9] S. Faridi, The facet ideal of a simplicial complex, Manuscripta Math. 109 (2002), 159-174.

[10] S. Faridi, Cohen-Macaulay properties of square-free monomial ideals, J. Combin. Theory Ser. A 109(2) (2005), 299-329.

[11] R. Fröberg, A study of graded extremal rings and of monomial rings, Math. Scand. 51 (1982), 22-34.

[12] R. Gilmer, Commutative Semigroup Rings, Chicago Lectures in Math., Univ. of Chicago Press, Chicago, 1984.

[13] I. Gitler, E. Reyes and R. H. Villarreal, Blowup algebras of ideals of vertex covers of bipartite graphs, Contemp. Math. 376 (2005), 273-279.

[14] I. Gitler, E. Reyes and R. H. Villarreal, Blowup algebras of square-free monomial ideals and some links to combinatorial optimization problems, Rocky Mountain J. Math., 39 (2009), no. 1, 71-102.

[15] I. Gitler, C. Valencia and R. H. Villarreal, A note on Rees algebras and the MFMC property, Beiträge Algebra Geom. 48 (2007), No. 1, 141-150.

[16] H. T. Hà, S. Morey and R.H. Villarreal, Cohen-Macaulay admissible clutters, Journal of Commutative Algebra, to appear.

[17] J. Herzog, T. Hibi, N. V. Trung and X. Zheng, Standard graded vertex cover algebras, cycles and leaves, Trans. Amer. Math. Soc. 360 (2008), 6231-6249.

[18] C. Huneke, A. Simis and W. V. Vasconcelos, Reduced normal cones are domains, Contemp. Math. 88 (1989), 95-101.

[19] C. Huneke and I. Swanson, Integral Closure of Ideals Rings, and Modules, London Math. Soc., Lecture Note Series 336, Cambridge University Press, Cambridge, 2006.

[20] A. Lehman, On the width-length inequality and degenerate projective planes, in Polyhedral Combinatorics (W. Cook and P. Seymour Eds.) DIMACS Series in 
Discrete Mathematics and Theoretical Computer Science 1, Amer. Math. Soc., 1990, pp. 101-105.

[21] H. Matsumura, Commutative Ring Theory, Cambridge Studies in Advanced Mathematics 8, Cambridge University Press, 1986.

[22] S. Morey, E. Reyes and R. H. Villarreal, Cohen-Macaulay, shellable and unmixed clutters with a perfect matching of König type, J. Pure Appl. Algebra 212(7)(2008), 1770-1786.

[23] A. Schrijver,Combinatorial Optimization, Algorithms and Combinatorics 24, Springer-Verlag, Berlin, 2003.

[24] A. Simis, W. V. Vasconcelos and R. H. Villarreal, On the ideal theory of graphs, J. Algebra, 167 (1994), 389-416.

[25] R. P. Stanley, Combinatorics and Commutative Algebra. Second edition. Progress in Mathematics 41. Birkhäuser Boston, Inc., Boston, MA, 1996.

[26] C. Valencia and R. H. Villarreal, Canonical modules of certain edge subrings, European J. Combin. 24(5) (2003), 471-487.

[27] A. Van Tuyl and R. H. Villarreal, Shellable graphs and sequentially CohenMacaulay bipartite graphs, J. Combin. Theory Ser. A 115 (2008), no.5, 799-814.

[28] W. V. Vasconcelos, Integral Closure, Springer Monographs in Mathematics, Springer, New York, 2005.

[29] R. H. Villarreal, Cohen-Macaulay graphs, Manuscripta Math. 66 (1990), 277293.

[30] R. H. Villarreal, Monomial Algebras, Monographs and Textbooks in Pure and Applied Mathematics 238, Marcel Dekker, New York, 2001.

[31] R. H. Villarreal, Rees algebras and polyhedral cones of ideals of vertex covers of perfect graphs, J. Algebraic Combin. 27(3) (2008), 293-305.

[32] X. Zheng, Resolutions of facet ideals, Comm. Algebra 32(6) (2004), 2301-2324. 